Journal of Agricultural Sciences
(Tarim Bilimleri Dergisi)

\title{
Palynological Study on Some Grape (Vitis vinifera L.) Cultivars Using Scanning Electron Microscopy
}

\author{
Burçak İş̧̧ía (iD)

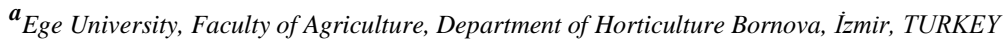 \\ ARTICLE INFO \\ Research Article \\ Corresponding Author: E-mail: burcak.isci@ege.edu.tr \\ Received: 14 April 2020 / Revised: 14 September 2020 / Accepted: 22 September 2020 / Online: 20 January 2022
}

\section{ABSTRACT}

Pollen is an important morphological parameter for cultivar identification. This is of great importance in detailed investigations by scanning electron microscope (SEM). In this study, the pollen morphology of selected grape cultivars was examined by SEM. The pollen length, width, P/E ratio of pollen and features of surface were observed. The pollen differed in some microstructural characteristic. Pollen width exhibited significant according to the varieties (10.12-22.44 $\mu \mathrm{m})$. Similarly, the statistical difference occurred among the thirty Vitis cultivars in terms of mean pollen length $(16.26-29.65 \mu \mathrm{m})$. Areolat pollen was determined in some cultivars. Depending on the cultivars there was significant differences in terms of pores diameter. According to PCA performed in 30 grape cultivars, 3 principal components were revealed and they defined $94.98 \%$ of the variance. Cultivars were divided into groups according to pollen features on the cluster. Consequently, the cultivars were categorized under two main groups. The present research is a contribution to a more detailed analysis of grapevine cultivars.

Keywords: Pollen, Morphology, Scanning electron microscope (SEM), Palynology, Classification, Description

(C) Ankara University, Faculty of Agriculture

\section{Introduction}

Grapes are most widely grown fruit crop. The grape is a member of the Vitaceae commonly called the grape family, Vitis contains around 60 species, plus some natural interspecific hybrids, and they are mostly distributed in temperate areas throughout the world (Mebberely 1987; Lombardi et al. 2007; Najmaddin et al. 2013). The grapes are one of the most important produce from the enological and economical point of view in the Anatolia. Generally, ampelographic studies have focused on the morphology of Vitis varieties. Pollen has hereditary properties that determine genotype. Pollen morphology confirms phylogenetic relationships among genera, species and varieties. Thus, it is used in systematic studies regarding to similarity and diversity of pollen. The morphology of pollen can be examined in detail via the scanning electron microscope (Tanaka et al. 2004).

On the basis of surface ornamentation and pollen grain dimensions, different classifications have been made on various plant species, such as grapevines (Wodehouse 1935; Erdtman 1952; Hyde \& Adams 1958; Faegri \& Iversen 1989). In the description of the pollen, qualitative characters, such as exine microrelief, separate elements, and quantitative characters, such as polar axis, equatorial axis, mesocolpium, apocolpium, and length and width of the colps are used (Roytchev 1995). For example, Uzun \& llter (1987) and Kharitonashyili et al. (1989) studied pollen grains in different types of flowers of Vitis vinifera L., using scanning electron microscopy (SEM). Ahmedullah (1983) characterized different grape cultivars based on pollen morphology. Martens et al. (1989) studied pollen size variability within genotypes of Vitis. Slimane \&Askri (1990) characterized 30 grapevine varieties based on pollen size, Roytchev et al. (1994) obtained information on the ultrastructure of exine surface apertures in 27 Bulgarian and repetition seedless grape cultivars. Palynology has presented considerable opportunities for some indigenous grape cultivars identification in grapevines, besides its importance in plant taxonomy (Marasalı et al. 2005). Gallardo et al. (2009) studied 14 Spanish Vitis vinifera L. subsp. sylvestris (Gmelin) Hegi populations. Jovanovic-Cvetkovic et al. (2016) analysed the pollen morphology of indigenous cvs. Žilavka and Blatina to determine their morphological specificities.

The objective of the present study was to classify the thirty grape cultivars according to the shape and microrelief of pollen grains and to establish the possibilities for using the parameters of the different apertures as classification indices using scanning electron microscope (SEM). 


\section{Material and Methods}

Pollen samples. This study was carried with pollen from thirty different of Vitis vinifera L. are located at the Department of Horticulture, Agriculture Faculty, Ege University, İzmir, Turkey (Table 1). The inflorescence was isolated. The pollen grains were gathered after these inflorescences were collected at the morning hours at the beginning of the blooming period (Eichhorn $\&$ Lorenz 1977). The pollen was sampled by cutting flowers and brushing the anthers and pollen into an Eppendorf tube using a soft brush (Gökbayrak \& Engin 2016). The pollen was stored until analysis (Storey 1975).

\section{Table 1- List of the cultivars studied}

\begin{tabular}{|c|c|c|c|c|c|c|c|c|c|}
\hline Cultivars & Type* & Cultivars & Type* & Cultivars & Type* & Cultivars & Type* & Cultivars & Type* \\
\hline $\begin{array}{l}\text { Vitis vinifera L. } \\
\text { "Abiguş" }\end{array}$ & $\mathrm{T}$ & $\begin{array}{l}\text { Vitis vinifera L. } \\
\text { "Alphonse } \\
\text { Lavallée'" }\end{array}$ & $\mathrm{T}$ & $\begin{array}{l}\text { Vitis vinifera L. } \\
\text { "Alicante } \\
\text { Bouschet" }\end{array}$ & W & $\begin{array}{l}\text { Vitis vinifera L. } \\
\text { "Beyaz Şam" }\end{array}$ & $\mathrm{T}$ & $\begin{array}{l}\text { Vitis vinifera L. } \\
\text { "Buca Razak1" }\end{array}$ & $\mathrm{T}$ \\
\hline $\begin{array}{l}\text { Vitis vinifera L. } \\
\text { "Cardinal" }\end{array}$ & $\mathrm{T}$ & $\begin{array}{l}\text { Vitis vinifera L. } \\
\text { "Cinsault" }\end{array}$ & W & $\begin{array}{l}\text { Vitis vinifera L. } \\
\text { "Çeşme } \\
\text { Pembesi" }\end{array}$ & $\mathrm{T}$ & $\begin{array}{l}\text { Vitis vinifera L. } \\
\text { "Foça Karası" }\end{array}$ & W & $\begin{array}{l}\text { Vitis vinifera L. } \\
\text { "Hafizali" }\end{array}$ & $\mathrm{T}$ \\
\hline $\begin{array}{l}\text { Vitis vinifera } \mathrm{L} \text {. } \\
\text { "Italia" }\end{array}$ & $\mathrm{T}$ & $\begin{array}{l}\text { Vitis vinifera } \mathrm{L} . \\
\text { "Kırmızı Şam }\end{array}$ & $\mathrm{T}$ & $\begin{array}{l}\text { Vitis vinifera L. } \\
\text { "Kozak } \\
\text { Gemresi" }\end{array}$ & $\mathrm{T}$ & $\begin{array}{l}\text { Vitis vinifera L. } \\
\text { "Mahrabaş1" }\end{array}$ & $\mathrm{T}$ & $\begin{array}{l}\text { Vitis vinifera L. } \\
\text { "Morsleleh }\end{array}$ & $\mathrm{T}$ \\
\hline $\begin{array}{l}\text { Vitis vinifera } \mathrm{L} \text {. } \\
\text { "Malbec" }\end{array}$ & W & $\begin{array}{l}\text { Vitis vinifera } \mathrm{L} \text {. } \\
\text { "Morseyhative" }\end{array}$ & $\mathrm{T}$ & $\begin{array}{l}\text { Vitis vinifera } \mathrm{L} \text {. } \\
\text { "Müşküle" }\end{array}$ & $\mathrm{T}$ & $\begin{array}{l}\text { Vitis vinifera L. } \\
\text { "Papaz Karası" }\end{array}$ & W & $\begin{array}{l}\text { Vitis vinifera } \mathrm{L} \text {. } \\
\text { "Pembe } \\
\text { Gemre" }\end{array}$ & $\mathrm{T}$ \\
\hline $\begin{array}{l}\text { Vitis vinifera L. } \\
\text { "Pek Üzümü" }\end{array}$ & $\mathrm{T}$ & $\begin{array}{l}\text { Vitis vinifera L. } \\
\text { "Siyah Gemre" }\end{array}$ & $\mathrm{T}$ & $\begin{array}{l}\text { Vitis vinifera L. } \\
\text { "Syrah" }\end{array}$ & W & $\begin{array}{l}\text { Vitis vinifera } \mathrm{L} \text {. } \\
\text { "Şika" }\end{array}$ & $\mathrm{T}$ & $\begin{array}{l}\text { Vitis vinifera L. } \\
\text { "Tarsus } \\
\text { Pembesi" }\end{array}$ & $\mathrm{T}$ \\
\hline $\begin{array}{l}\text { Vitis vinifera } \mathrm{L} \text {. } \\
\text { "Trakya } \\
\text { İlkeren" }\end{array}$ & $\mathrm{T}$ & $\begin{array}{l}\text { Vitis vinifera L. } \\
\text { "Öküzgözü" }\end{array}$ & W & $\begin{array}{l}\text { Vitis vinifera L. } \\
\text { "Ohannes" }\end{array}$ & $\mathrm{T}$ & $\begin{array}{l}\text { Vitis vinifera L. } \\
\text { "Yuvarlak } \\
\text { Çekirdeksiz" }\end{array}$ & $\mathrm{T}, \mathrm{SE}$ & $\begin{array}{l}\text { Vitis vinifera L. } \\
\text { "Yuvarlak } \\
\text { Razak1" }\end{array}$ & $\mathrm{T}$ \\
\hline
\end{tabular}

*T: table grape; W: vine grape; SE: seedless grape

Pollen grains were air dried for investigation in SEM. Dry pollen was sputter-coated (Leica model) with $10 \mu \mathrm{m}$ of goldpalladium. Pollens were measured directly on the screen of the electron microscope. Each of the tested samples were observed with scanning electron microscope (Thermo Scientific Apreo S model) were photographed at $10000 \mathrm{x}$ for whole grain. The pollen length, width, length/width ratio and pore diameter, distance between pores and colpi length were measured at 10 pollen grains for each genotypes. The pollen shape was stated by considering the length/width ratio (Erdtman 1952). The types of aperture found in pollen were described according to Wang et al. (2014). The polar (P) and equatorial (E) axes, P/E relationship were determined according to Van der Pluym \& Hideux (1977). The terminology of Erdman (1952) was used in the morphological descriptions of the pollen.

The data were subjected to analysis of variance using SPSS (SSPS Inc. 10.0, USA, 1999) statistical package program. The differences between the means were determined with Fischer's Least Significant Difference (LSD) test. The mean, minimum, maximum" and standard deviation values of the properties were found out. These values were revealed by conducting Pearson's correlation analysis. Further, Principal Component Analysis (PCA) and Clustering Analysis (CA) were also performed and indicated by dendrogram. Differences or similarities of cultivars were evaluated according to their analyzed properties by applying PCA to the findings obtained. Moreover, cluster analysis was utilized to create a dendrogram showing similarities and differences between genotypes.

\section{Results and Discussion}

Given the characteristics of the thirty grape cultivars, a general description was established for all, according to the values of the various parameters corresponding to the max. and min. records of the cultivars. The statistical difference appeared among the thirty different of Vitis vinifera L. genotypes in terms of the pollen grains (pollen length, width and length/width ratio). The variation in the min., max. mean values and standard deviations of grape pollen parameters are shown in Table 2 and Table 3.

Mean pollen width differed statistically significant among the varieties. Thus, the highest mean values for this feature were determined on "Yuvarlak Çekirdeksiz" $(19.09 \mu \mathrm{m})$ and "Mahrabaşı" $(22.44 \mu \mathrm{m})$ and the lowest mean values for this pollen width were found in "Alicante Bouschet" $(10.12 \mu \mathrm{m})$, "Öküzgözü" $(10.32 \mu \mathrm{m})$ and "Syrah" (10.54 $\mu \mathrm{m})$ varieties respectively. Pollen length ranged from $29.65 \mu \mathrm{m}$ "Alphonse Lavallée"” to $16.26 \mu \mathrm{m}$ "Foça Karası". As followed, differences in "Alphonse Lavallée" genotype (28.28 to $32.32 \mu \mathrm{m})$ caused to a higher standard deviation (1.50) (Table 2). 
Table 2- Morphological characteristic of pollen of grape cultivars $(\mu \mathrm{m})$

\begin{tabular}{|c|c|c|c|c|c|c|c|c|c|c|c|c|}
\hline \multirow{3}{*}{ Cultivars } & \multicolumn{12}{|c|}{ Mean Pollen Size } \\
\hline & \multicolumn{4}{|c|}{ Pollen length $(\mu \mathrm{m})$} & \multicolumn{4}{|c|}{ Pollen width $(\mu \mathrm{m})$} & \multicolumn{4}{|c|}{ Length/width ratio } \\
\hline & Min & $\operatorname{Max}$ & Mean & $S D$ & Min & $\operatorname{Max}$ & Mean & $S D$ & Min & $\operatorname{Max}$ & Mean & $S D$ \\
\hline 1. & 15.70 & 17.26 & $16.26 \mathrm{~m}$ & 0.62 & 14.17 & 16.89 & 15.04 fghi & 0.94 & 1.02 & 1.13 & $1.08 \mathrm{n}$ & 0.04 \\
\hline 2. & 23.55 & 29.87 & 26.86 abcdefgh & 2.12 & 20.82 & 24.25 & $22.44 \mathrm{a}$ & 1.03 & 1.08 & 1.39 & $1.20 \mathrm{mn}$ & 0.13 \\
\hline 3. & 27.12 & 31.43 & $28.34 \mathrm{abcd}$ & 1.30 & 15.07 & 21.43 & $16.54 \mathrm{cdef}$ & 1.86 & 1.47 & 1.81 & 1,72 hijk & 0.10 \\
\hline 4. & 22.15 & 26.46 & 23.97 hijkl & 1.40 & 16.42 & 19.10 & $17.39 \mathrm{bc}$ & 0.68 & 1.16 & 1.61 & $1.38 \mathrm{~lm}$ & 0.12 \\
\hline 5. & 26.47 & 30.56 & 28.18 abcde & 1.61 & 15.28 & 18.89 & $17.70 \mathrm{bc}$ & 1.01 & 1.42 & 1.75 & $1.60 \mathrm{ijkl}$ & 0.14 \\
\hline 6. & 19.86 & 26.99 & 25.32 efghij & 2.13 & 12.09 & 18.58 & $16.20 \mathrm{cdef}$ & 1.87 & 1.41 & 1.65 & $1.57 \mathrm{kl}$ & 0.08 \\
\hline 7. & 28.28 & 32.32 & $29.65 \mathrm{a}$ & 1.50 & 14.50 & 16.76 & 15.55 efgh & 0.70 & 1.75 & 2.08 & 1.91 cdefghi & 0.13 \\
\hline 8. & 19.23 & 30.05 & 26.99 abcdefg & 3.45 & 13.11 & 16.96 & 14.72 fghij & 1.21 & 1.44 & 2.15 & 1.84 efghijk & 0.23 \\
\hline 9. & 24.34 & 32.86 & $28.42 \mathrm{abc}$ & 2.64 & 14.06 & 17.00 & $15.90 \mathrm{cdef}$ & 1.18 & 1.50 & 2.01 & 1.79 fghijk & 0.15 \\
\hline 10. & 17.89 & 28.57 & 22.161 & 3.50 & 11.78 & 22.08 & 14.56 fghij & 3.01 & 1.09 & 2.02 & $1.56 \mathrm{kl}$ & 0.33 \\
\hline 11. & 21.43 & 29.02 & 25.43 defghi & 2.69 & 8.18 & 13.07 & 10.121 & 1.49 & 2.09 & 3.35 & $2.55 \mathrm{a}$ & 0.41 \\
\hline 12. & 22.79 & 29.10 & 25.98 cdefghi & 1.98 & 10.07 & 16.48 & $12.98 \mathrm{jk}$ & 2.32 & 1.66 & 2.50 & 2.04 cdefg & 0.26 \\
\hline 13. & 20.44 & 26.11 & $22.59 \mathrm{jkl}$ & 1.95 & 9.59 & 18.56 & $12.77 \mathrm{jk}$ & 2.56 & 1.41 & 2.44 & 1.82 efghijk & 0.36 \\
\hline 14. & 20.82 & 29.52 & 25.74 cdefghi & 2.99 & 9.65 & 17.03 & $12.94 \mathrm{jk}$ & 2.79 & 1.46 & 2.95 & 2.08 cdef & 0.54 \\
\hline 15. & 20.25 & 31.90 & 25.04 ghijk & 3.46 & 9.11 & 12.28 & 10.541 & 0.97 & 1.70 & 3.06 & $2.40 \mathrm{ab}$ & 0.39 \\
\hline 16. & 17.21 & 30.71 & $22.48 \mathrm{kl}$ & 4.25 & 9.20 & 11.96 & 10.321 & 0.96 & 1.59 & 3.25 & $2.21 \mathrm{bc}$ & 0.54 \\
\hline 17. & 18.61 & 32.13 & 27.19 abcdefg & 4.09 & 12.41 & 20.23 & 15.10 fghi & 2.57 & 1.31 & 2.32 & 1.84 efghijk & 0.37 \\
\hline 18. & 20.31 & 30.47 & 25.13 fghijk & 3.77 & 12.97 & 19.42 & 15.67 defgh & 2.60 & 1.19 & 2.24 & $1.64 \mathrm{ijkl}$ & 0.34 \\
\hline 19. & 21.83 & 30.91 & 25.68 cdefghi & 2.69 & 11.55 & 15.65 & 13.74 hijk & 1.09 & 1.68 & 2.29 & 1.88defghijk & 0.22 \\
\hline 20. & 19.23 & 28.47 & 25.01 ghijk & 3.03 & 11.24 & 16.36 & $12.74 \mathrm{jk}$ & 1.76 & 1.62 & 2.47 & 1.99 cdefgh & 0.31 \\
\hline 21. & 21.67 & 29.02 & 26.53 cdefghi & 2.29 & 11.29 & 17.73 & 14.17 ghijk & 2.08 & 1.56 & 2.29 & 1.90 cdefghij & 0.24 \\
\hline 22. & 22.00 & 29.89 & 25.57 cdefghi & 2.36 & 10.38 & 16.27 & $12.69 \mathrm{jk}$ & 1.85 & 1.49 & 2.38 & 2.05 cdefg & 0.28 \\
\hline 23. & 20.38 & 28.01 & 25.49 defghi & 2.19 & 10.13 & 14.19 & $12.26 \mathrm{k}$ & 1.65 & 1.74 & 2.76 & 2.11 bcde & 0.33 \\
\hline 24. & 18.96 & 33.25 & 28.13 abcde & 4.28 & 14.00 & 20.74 & $17.61 \mathrm{bc}$ & 2.06 & 1.19 & 1.96 & $1.60 \mathrm{ijkl}$ & 0.23 \\
\hline 25. & 20.84 & 27.40 & $23.67 \mathrm{ijkl}$ & 2.52 & 11.01 & 18.35 & 13.86 hijk & 2.64 & 1.29 & 2.13 & 1,75 ghijk & 0.27 \\
\hline 26. & 26.98 & 32.27 & $29.43 \mathrm{ab}$ & 1.74 & 11.43 & 16.90 & 13.96 ghijk & 1.74 & 1.78 & 2.60 & 2.14 bcde & 0.27 \\
\hline 27. & 26.98 & 32.27 & $29.43 \mathrm{ab}$ & 1.74 & 11.43 & 16.90 & 14.02 ghijk & 1.82 & 1.71 & 2.60 & 2.13 bcde & 0.28 \\
\hline 28. & 24.16 & 31.30 & 28.04 abcdef & 2.72 & 10.85 & 16.74 & $13.32 \mathrm{ijk}$ & 1.94 & 1.59 & 2.74 & $2.16 \mathrm{bcd}$ & 0.43 \\
\hline 29. & 23.19 & 34.47 & 26.47 cdefghi & 3.37 & 14.83 & 23.81 & $19.09 \mathrm{a}$ & 3.15 & 1.03 & 1.67 & $1.42 \mathrm{~lm}$ & 0.26 \\
\hline 30. & 22.76 & 29.02 & 26.60 bcdefgh & 1.97 & 11.79 & 21.58 & $17.18 \mathrm{cdef}$ & 2.64 & 1.26 & 2.46 & $1.59 \mathrm{jkl}$ & 0.30 \\
\hline
\end{tabular}

* Min: minimum values; Max: maximum values; SD: standard deviations; 1. Vitis vinifera L. "Foça Karası"; 2. Vitis vinifera L. "Mahrabaş"; 3. Vitis vinifera

L. "Yuvarlak Razakı"; 4. Vitis vinifera L. "Beyaz Şam"; 5. Vitis vinifera L. "Müşküle”; 6. Vitis vinifera L. "Trakya İlkeren”; 7. Vitis vinifera L. "Alphonse Lavallée"”; 8. Vitis vinifera L. "Siyah Gemre"; 9. Vitis vinifera L. "Cinsault"; 10. Vitis vinifera L. "Kozak Gemresi"; 11. Vitis vinifera L. "Alicante

Bouschet"; 12. Vitis vinifera L. "Buca Razak1"; 13. Vitis vinifera L. "Malbec"; 14. Vitis vinifera L. "İtalia"; 15. Vitis vinifera L. "Syrah", 16. Vitis vinifera L. "Öküzgözü”, 17. Vitis vinifera L. "Pembe Gemre”, 18. Vitis vinifera L. "Ohannes”, 19. Vitis vinifera L. "Papaz Karası", 20. Vitis vinifera L. "Kırmızı Şam”,

21. Vitis vinifera L. "Morseyhative", 22. Vitis vinifera L. "Morsleleh", 23. Vitis vinifera L. "Abiguş", 24. Vitis vinifera L. "Hafizali", 25. Vitis vinifera L.

"Çeşme Pembesi”, 26. Vitis vinifera L. "Cardinal”, 27. Vitis vinifera L. "Pek Üzümü”, 28. Vitis vinifera L. "Tarsus Pembesi”, 29. Vitis vinifera L. "Yuvarlak Çekirdeksiz", 30. Vitis vinifera L.

On the other hand, when the pollen is examined in terms of symmetry and shape, the length/width ratio ranged from $2.55 \mu \mathrm{m}$ "Alicante Bouschet" to 1.08 "Foça Karası" (Table 2). The terminology of Erdman (1952) was used in the morphological descriptions of the pollen. The pollen grains were prolate-spheroidal "Foça Karası" (8:7-8:8), subprolate "Mahrabaşı" (7:8-6:8), perprolate (“Alicante Bouschet”, "Syrah”, “Öküzgözü”, "Tarsus Pembesi”, “Abiguş”, “Cardinal”, 
"Pek Üzümü", “Italia", "Morsleleh", "Buca Razakı" (>8:4)) and prolate ("Kırmızı Şam”, "Alphonse Lavallée’”, "Morseyhative", "Papaz Karası", "Siyah Gemre”, "Pembe Gemre”, "Malbec”, "Cinsault”, "Çeşme Pembesi”, "Yuvarlak Razakı", "Müş̧üle”, “Ohannes”, "Hafızali”, “Şika”, “Trakya İlkeren”, Kozak Gemresi”, "Yuvarlak Çekirdeksiz”, "Beyaz Şam” (8:4-8:6)) (Figure 1).

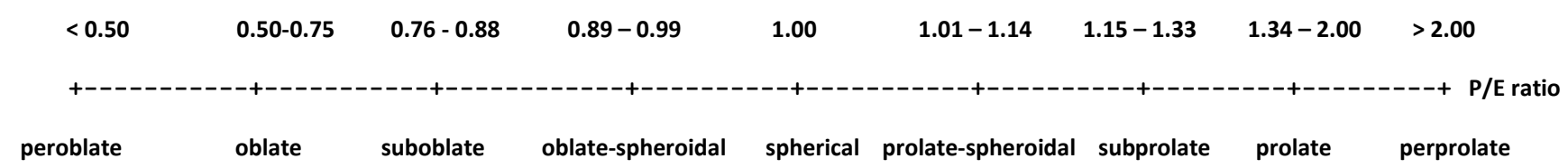

Figure 1- Mean value for P/E ratio (Marasalı et al. 2005; Gökbayrak \& Engin 2016)

According to aperture, typically two types were observed. Among the grape varieties examined, it was determined that there was no diaphragm opening in the pollen of a group. Inaperturate pollen grains were observed in some cultivars such as "Foça Karası", "Mahrabaşı", "Trakya İlkeren", "Kozak Gemresi”, "Ohannes” and "Çeşme Pembesi”, whereas "Yuvarlak Razakı", "Beyaz Şam”, "Müşküle”, “Alphonse Lavallée”, "Siyah Gemre”, "Cinsault”, “Alicante Bouschet”, "Buca Razakı”, "Malbec”, "İtalia”, "Syrah" “Öküzgözü”, "Pembe Gemre”, "Papaz Karası”, "Kırmızı Şam”, "Morseyhative”, "Morsleleh”, “Abiguş", "Hafizali", "Çeşme Pembesi”, "Cardinal”, "Pek Üzümü”, "Tarsus Pembesi”, "Yuvarlak Çekirdeksiz”, and "Şika" were tricolporate (Figure 2).
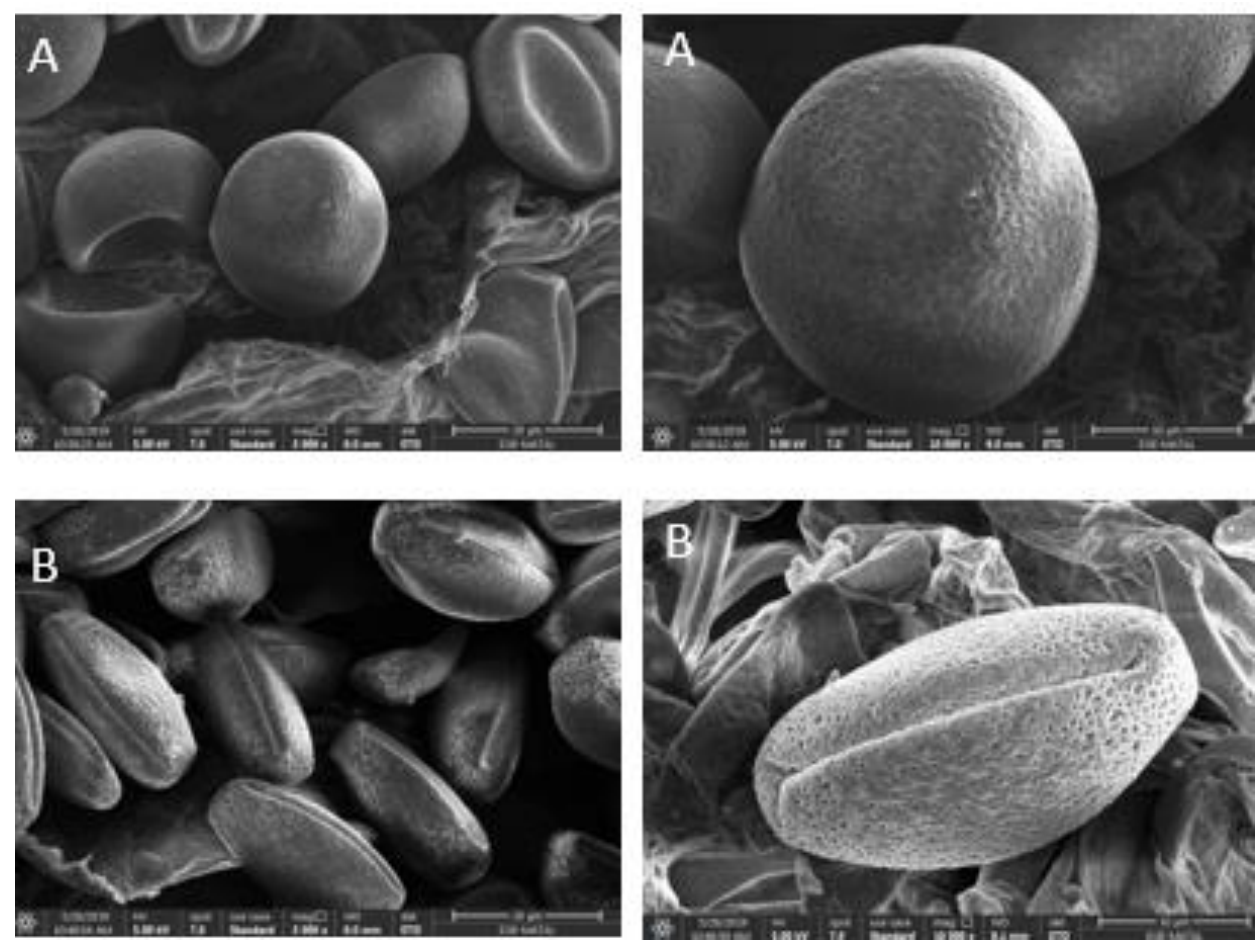

Figure 2- Scanning electron microscope image of pollen A: Inaperturate pollen B: Tricolporate pollen

Circular openings were detected on the pollen grains and such grains are called porate. The pollen had circular apertures on the exine surface, these were not uniformly distributed and the pollen grains were said pantoporate. The pollen grains surface has elongated or furrow-like apertures. These were called colp. Also, the circular apertures on the pollen has circular apertures on the exine surface, they were called pores. The pollen shape and exine patterns of the studied varieties were given in SEM images. There were statistical differences in terms of these properties. For pores length, "Pek Üzümü" (512.29) located at the first group, while the "Alphonse Lavallée"” (112.45) was the last group. Thus, pores width differed statistically significant according to the varieties. For this value, the "Morseyhative" (435.13), "Tarsus Pembesi" (405.98), "Çeşme Pembesi” (405.76) and "Trakya İlkeren" (389.33) varieties were the first group, among the varieties examined, "Syrah" was the smallest diameter of the pores width and, "Syrah" (97.06) located at the last group. In terms of this feature, it was found in different statistical groups in other varieties (Table 3). 
Table 3- The length and width values of pores in grape varieties $(\mu \mathrm{m})$

\begin{tabular}{|c|c|c|c|c|c|c|c|c|}
\hline \multirow{2}{*}{ Cultivars } & \multicolumn{4}{|c|}{ Pores length $(\mu \mathrm{m})$} & \multicolumn{4}{|c|}{ Pores width ( $\mu \mathrm{m})$} \\
\hline & Min & $\operatorname{Max}$ & Mean & $S D$ & Min & $\operatorname{Max}$ & Mean & $S D$ \\
\hline 1. & 152.91 & 276.75 & $204.06 \mathrm{efg}$ & 51.24 & 141.95 & 260.77 & 196.85 cdefg & 48.74 \\
\hline 2. & 109.80 & 332.63 & 208.54 efg & 111.45 & 149.30 & 315.66 & $220.11 \mathrm{bcdef}$ & 70.65 \\
\hline 3. & 103.26 & 373.89 & 221.16 defg & 120.53 & 106.60 & 193.19 & $136.22 \mathrm{efg}$ & 33.33 \\
\hline 4. & 321.56 & 482.35 & $396.57 \mathrm{abcd}$ & 66.87 & 159.84 & 498.23 & $328.72 \mathrm{ab}$ & 141.31 \\
\hline 5. & 144.56 & 280.39 & $186.67 \mathrm{fg}$ & 55.82 & 134.75 & 225.46 & 161.38 cdefg & 36.37 \\
\hline 6. & 265.00 & 502.46 & $411.81 \mathrm{abc}$ & 91.41 & 250.00 & 483.65 & $389.33 \mathrm{a}$ & 93.90 \\
\hline 7. & 99.46 & 146.52 & $112.45 \mathrm{~g}$ & 20.15 & 84.91 & 135.83 & $102.32 \mathrm{fg}$ & 20.13 \\
\hline 8. & 181.26 & 398.55 & 291.52 cdefg & 78.13 & 166.76 & 289.55 & 247.52 bcde & 47.16 \\
\hline 9. & 322.00 & 492.54 & $397.45 \mathrm{abcd}$ & 71.91 & 124.56 & 176.55 & 158.09 defg & 22.20 \\
\hline 10. & 159.94 & 293.33 & 208.50 efg & 51.79 & 156.54 & 267.45 & 195.59 cdefg & 44.84 \\
\hline 11. & 194.20 & 280.33 & 217.18 defg & 35.64 & 167.56 & 200.18 & 185.69 cdefg & 16.11 \\
\hline 12. & 167.40 & 851.10 & $413.34 \mathrm{abc}$ & 276.47 & 150.80 & 510.30 & $280.92 \mathrm{bc}$ & 135.39 \\
\hline 13. & 217.20 & 349.90 & 298.82 bcdefg & 52.02 & 180.51 & 250.54 & 209.07 cdefg & 25.73 \\
\hline 14. & 159.30 & 305.50 & $219.18 \mathrm{defg}$ & 63.19 & 94.86 & 136.80 & $118.63 \mathrm{fg}$ & 21.30 \\
\hline 15. & 111.20 & 288.90 & $178.48 \mathrm{fg}$ & 75.82 & 86.00 & 133.50 & $97.06 \mathrm{~g}$ & 20.43 \\
\hline 16. & 99.49 & 356.50 & 228.46 defg & 108.11 & 80.68 & 235.60 & $121.91 \mathrm{fg}$ & 63.99 \\
\hline 17. & 113.30 & 372.50 & 218.54 defg & 114.11 & 100.80 & 190.16 & $134.22 \mathrm{efg}$ & 33.32 \\
\hline 18. & 149.90 & 493.20 & 330.24 bcdef & 146.35 & 135.23 & 350.21 & 196.34 cdefg & 90.53 \\
\hline 19. & 118.80 & 347.20 & $213.46 \mathrm{defg}$ & 115.16 & 115.55 & 135.66 & $160.39 \mathrm{defg}$ & 79.70 \\
\hline 20. & 176.20 & 978.20 & 384.28 abcde & 334.34 & 84.98 & 267.80 & 189.01 cdefg & 68.75 \\
\hline 21. & 197.60 & 778.70 & $474.54 \mathrm{ab}$ & 282.97 & 150.11 & 770.80 & $435.13 \mathrm{a}$ & 270.89 \\
\hline 22. & 214.90 & 354.70 & 311.98 bcdef & 56.52 & 180.66 & 340.91 & $272.57 \mathrm{bcd}$ & 73.78 \\
\hline 23. & 141.90 & 217.50 & $175.20 \mathrm{fg}$ & 27.92 & 120.37 & 177.80 & $155.79 \mathrm{defg}$ & 24.60 \\
\hline 24. & 142.90 & 270.70 & $198.57 \mathrm{efg}$ & 51.63 & 140.05 & 166.35 & $152.57 \mathrm{defg}$ & 11.09 \\
\hline 25. & 419.80 & 439.20 & $430.93 \mathrm{abc}$ & 7.83 & 386.51 & 421.35 & $405.76 \mathrm{a}$ & 16.57 \\
\hline 26. & 178.39 & 398.80 & 265.36 cdefg & 91.58 & 165.72 & 281.35 & $245.68 \mathrm{bcde}$ & 46.05 \\
\hline 27. & 498.65 & 546.65 & $512.29 \mathrm{a}$ & 19.54 & 191.60 & 203.63 & 198.14 cdefg & 4.33 \\
\hline 28. & 291.00 & 523.66 & $431.81 \mathrm{abc}$ & 113.64 & 280.00 & 498.70 & $405.98 \mathrm{a}$ & 98.63 \\
\hline 29. & 147.30 & 295.60 & 214.60 defg & 57.80 & 135.81 & 228.64 & 161.62 cdefg & 37.84 \\
\hline 30. & 188.50 & 333.30 & $232.32 \mathrm{defg}$ & 61.57 & 170.80 & 328.74 & 212.80 cdefg & 65.79 \\
\hline
\end{tabular}

Min: minimum values; Max: maximum values; SD: standard deviations ;1. Vitis vinifera L. "Foça Karası", 2. Vitis vinifera L. "Mahrabaşı", 3. Vitis vinifera L. "Yuvarlak Razakı", 4. Vitis vinifera L. "Beyaz Şam”, 5. Vitis vinifera L. "Müşküle”, 6. Vitis vinifera L. "Trakya İlkeren”, 7. Vitis vinifera L. "Alphonse Lavallée"”, 8. Vitis vinifera L. "Siyah Gemre", 9. Vitis vinifera L. "Cinsault", 10. Vitis vinifera L. "Kozak Gemresi", 11. Vitis vinifera L. "Alicante Bouschet", 12. Vitis vinifera L. "Buca Razakı", 13. Vitis vinifera L. "Malbec", 14. Vitis vinifera L. "İtalia", 15. Vitis vinifera L. "Syrah", 16. Vitis vinifera L. "Öküzgözü”, 17. Vitis vinifera L. "Pembe Gemre”, 18. Vitis vinifera L. "Ohannes”, 19. Vitis vinifera L. "Papaz Karası", 20. Vitis vinifera L. "Kırmızı Şam”, 21. Vitis vinifera L. "Morseyhative”, 22. Vitis vinifera L. "Morsleleh", 23. Vitis vinifera L. "Abiguş", 24. Vitis vinifera L. "Hafizali”, 25. Vitis vinifera L. "Çeşme Pembesi”, 26. Vitis vinifera L. “Cardinal”, 27. Vitis vinifera L. "Pek Üzümü”, 28. Vitis vinifera L. "Tarsus Pembesi", 29. Vitis vinifera L. "Yuvarlak Çekirdeksiz", 30. Vitis vinifera L. "Şika"

Scrobiculate pollen was detected in "Alicante Bouschet", "Buca Razak1", "Ohannes", "Papaz Karası", "Morseyhative", "Morsleleh", "Hafizali", and "Tarsus Pembesi", on the other hand striate pollen was found among the other varieties (Figure 3). Pollen of some cultivars had not furrows. These pollens were found in "Alicante Bouschet", "Buca Razakı", "Syrah", "Ohannes", "Morseyhative", "Hafizali", "Cardinal”, "Tarsus Pembesi”, and "Şika" cultivars. Areolat was observed in "Foça Karası", "Mahrabaşı", "Yuvarlak Razakı”, "Beyaz Şam”, "Müşküle”, “Trakya İlkeren”, “Alphonse Lavallée”, "Siyah Gemre”, “Cinsault”, "Kozak Gemresi”, "Malbec”, "Italia”, "Öküzgözü”, "Pembe Gemre”, "Papaz Karası", "Kırmızı Şam”, "Morsleleh”, “Abiguş”, "Çeşme Pembesi”, "Pek Üzümü”, and "Yuvarlak Çekirdeksiz" (Figure 3). 

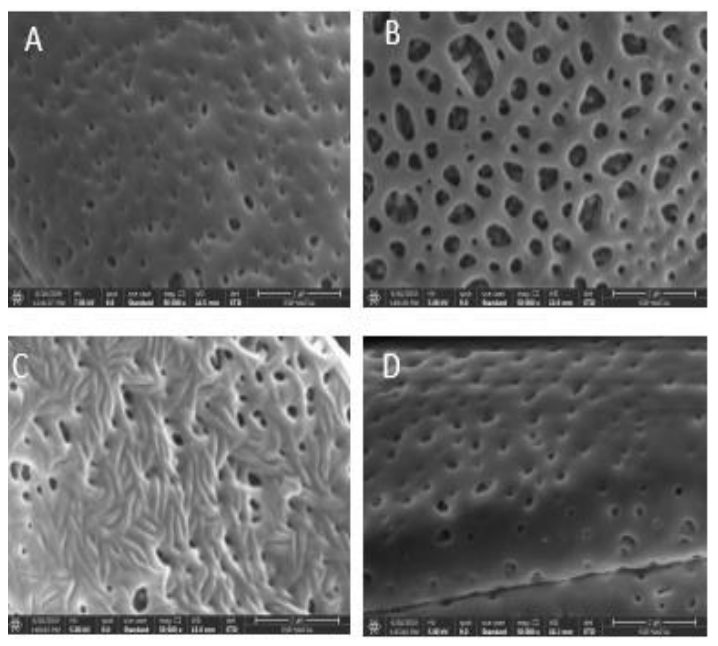

Figure 3- Pollen exine ornamentati on image A: Scrobiculate pollen B: Striate pollen C: Areola pollen D: without furrows pollen

The correlation coefficients of the features are shown in Table 4. Accordingly, the highest positive correlation was determined between pores length and pores width $(\mathrm{r}=0.739 ; \mathrm{P}<0.01)$. From the other side, a negative correlation occurred between the pollen length/width ratio and pollen width value $(\mathrm{r}=-0.816 ; \mathrm{P}<0.01)$. Correlation between pollen length/width ratio and pollen width is not meaningful.

Table 4- Pearson correlation coefficient among traits in cultivars

\begin{tabular}{lcccc}
\hline Traits & Pollen width & Pollen length & Pollen length/width ratio & Pores width \\
\hline Pollen length & 0.247 & & & \\
Pollen length/width ratio & $\mathbf{- 0 . 8 1 6}^{* *}$ & 0.315 & & \\
Pores width & 0.045 & -0.073 & 0.123 & $\mathbf{0 . 7 3 9}^{* *}$ \\
Pores length & 0.104 & 0.076 & 0.080 & \\
\hline
\end{tabular}

Abbreviations: * Significant at $\mathrm{P}<0.05 ; * *$ Significant at $\mathrm{P}<0.01$

Clustering analysis was used to determine the degree of similarity of grape cultivars, is located in Figure 4 as dendograms. Consequently, the cultivars were categorized under two main groups. "Yuvarlak Razakı", "Pembe Gemre”, "İtalia”, “Öküzgözü”, "Müşküle”, “Abiguş", "Papaz Karası", "Yuvarlak Çekirdeksiz”, “ Hafizali”, “Alphonse Lavallée”, "Syrah” "Foça Karası”, "Kozak Gemresi”, "Alicante Bouschet”, "Mahrabaşı”, "Şika” were included in the first group while "Beyaz Şam”, "Buca Razakı", "Çeşme Pembesi”, “Tarsus Pembesi”, “Trakya İlkeren”, "Morseyhative”, "Malbec”, "Ohannes”, "Siyah Gemre”, "Cardinal", "Morsleleh", "Cinsault”, "Kırmızı Şam”, and "Pek Üzümü”, were collected in the second group. First and second groups divided into different sub-groups. The similarity of pollens of grape cultivars examined with CA showed a correlation with those examined with PCA in terms of examined characteristics.

Table 5 shows the degree of similarity of grape varieties with clustering analysis. Therefore, the relationships between grape genotypes examined with CA. According to PCA performed in 30 grape genotypes, 3 principal components were revealed and they defined $94.98 \%$ of the variance (Table 5). In this way, pollen width and the length/width ratio had the highest positive contribution to PC1, constituting $36.68 \%$ of the total variance, which is the most important component. The pores length and between pores width contributed to PC2, accounting for $34.81 \%$ of the total variance. On the other hand, PC3 constituted pollen length accounting for $34.81 \%$ of the total variance (Table 5).

Table 5- Component loading in Principle Component Analysis (PCA)

\begin{tabular}{lrrr}
\hline Traits & $\boldsymbol{P C 1}$ & $\boldsymbol{P C 2}$ & $\boldsymbol{P C 3}$ \\
\hline Pollen width & $\mathbf{0 . 9 6 0}$ & -0.029 & 0.262 \\
Length/width ratio & $\mathbf{- 0 . 9 4 4}$ & -0.026 & 0.309 \\
Pores length & -0.105 & $\mathbf{0 . 9 3 3}$ & 0.086 \\
Pores width & 0.101 & $\mathbf{0 . 9 3 2}$ & -0.087 \\
Pollen length & -0.010 & 0.002 & $\mathbf{0 . 9 9 8}$ \\
Eigenvalue & 1.834 & 1.741 & 1.175 \\
Proportion (\%) & 36.674 & 34.813 & 34.813 \\
Cumulative (\%) & 36.674 & 71.487 & 94.977 \\
\hline
\end{tabular}

Principal Component Analysis. Rotation Method: Varimax with Kaiser Normalization 
Characterization of grape cultivars by pollen grains has been relatively frequent. A number of palynological investigations into cultivated Vitis varieties also showed that pollen shape and P/E ratio change from one sample to another (Reille 1966; Cabello et al. 1994; Roytchev 1997). Our results revealed that the thirty cultivars of $V$. vinifera exhibited differences about the pollen morphology. There were difference in the size (pollen width, pollen length), shape of pollen grains, pores on pollen surface and pollen ornamentation. All features reviewed were found to be the most important parameters for characterization.

There were significantly differences in pollen width grains sizes in the cultivars studied. The maximum pollen width size in the "Mahrabaşı" $(22.44 \mu \mathrm{m})$ and the highest in "Yuvarlak Çekirdeksiz" $(19.09 \mu \mathrm{m})$. The lenght of pollen grains ranged from $16.25 \mu \mathrm{m}$ (Foça Karası) to $29.65 \mu \mathrm{m}$ (Alphonse Lavallée'). Pollen width grains sizes of the "Cardinal” studied has $13.96 \mu \mathrm{m}$, and lenght of pollen grains has $29.43 \mu \mathrm{m}$. In relation to the results reported by Marasalı et al. (2005) and Gökbayrak \& Engin (2016), the "Cardinal" pollens were medium sized, the values obtained in our studies were higher than those of the mentioned studies. The pollen width and length of "Yuvarlak Çekirdeksiz" were $19.09 \mu \mathrm{m}$ and $26.27 \mu \mathrm{m}$, respectively. Roytchey et al. (1994) reported that pollen is same sized (the highest mean values - 24.13 and $24.04 \mu \mathrm{m}$ ) all investigated the seedless grape cultivars. As it is seen, it has been revealed that there are studies on pollen morphology in seedless grape cultivars.

Vitis is characterized by its 3-colporate grains. However, there was difference in pollen shape in our study. Prolate pollen grain was found in the material from "Foça Karası". Subprolate pollen grains was found in "Mahrabaşı". Perprolate pollen grains were on "Alicante Bouschet”, "Syrah”, “Öküzgözü”, "Tarsus Pembesi”, “Abiguş", “Cardinal”, "Pek Üzümü”, “Italia”, "Morsleleh", "Buca Razak1" and prolate were in "Kırmızı Şam”, “Alphonse Lavallée”, "Morseyhative”, "Papaz Karası”, "Siyah Gemre", "Pembe Gemre”, "Malbec”, "Cinsault”, "Çeşme Pembesi”, "Yuvarlak Razakı", "Müşküle”, "Ohannes”, "Hafizali”, "Şika”, "Trakya İlkeren”, "Kozak Gemresi”, "Yuvarlak Çekirdeksiz”, "Beyaz Şam”. To confirm our findings, pollen shape and P/E ratio differed from the findings of Marasalı et al. (2005) and Gökbayrak \& Engin (2016) for grapes. Roytchev (1997) reported in seedless cultivars, this ratio varies from 1.10 (cv. Seedless Red) to 2.08 (cv. Russalka), being $<2$ for most of the cultivars. The elliptical oval shape of pollen grains is typical for most of the seedless grapes. Inceoglu et al. (2000) stated that pollens of Vitis sylvestris ranged from prolate-spheroidal and subprolate pollen shape.

Erdtman (1952) reported reticulate pollen grains in members of the family Vitaceae. Faegri \& Iversen 1989, on the other hand, reported that the exine sculpturing of Vitis was reticulate, foveolate-perforate and that lumina size increased towards the poles under LM. This study showed that in the thirty grape cultivars, exine sculpturing was obscurely reticulate under SEM, and scrobiculate and striate at the mesocolpia and distinctly reticulate at and around the poles. An increase in lumina size towards the poles, observed by SEM, supports the results for Faegri \& Iversen (1989). The findings obtained by electron microscope in our study showed that the pollen morphological characteristics for the Vinifera cultivars can be used as a distinctive characteristic.

Ornamentation of the pollen is one of the most significant characteristics that can be used to separate cultivars. This situation reflects the variation between the cultivars. In grape species, the presence or absence furrows can be considered as useful tools for some taxonomic studies. Grape cultivars have also been subjected to palynological investigations (Reille 1966; Cabello et al. 1994; Roytchev 1997). As a result of this study, we found of furrows some of the examined cultivars, such pollens were found in "Alicante Bouschet", "Buca Razakı", "Syrah", "Ohannes”, "Morseyhative”, "Hafizali”, "Cardinal”, "Tarsus Pembesi”, "Şika" cultivars. Areolat is surrounded in exine were determined in cultivars of "Foça Karası", "Mahrabaşı", "Yuvarlak Razakı", "Beyaz Şam”, “Müşküle”, “Trakya İlkeren”, “Alphonse Lavallée”, "Siyah Gemre”, "Cinsault”, "Kozak Gemresi”, "Malbec”, "Italia”, “Öküzgözü”, "Pembe Gemre”, “Papaz Karası”, “Kırmızı Şam”, “Morsleleh”, “Abiguş”, “Çeşme Pembesi”, “Pek Üzümü”, "Yuvarlak Çekirdeksiz".

Pollen morphological characteristics such as pore structure, the ratio of $\mathrm{P} / \mathrm{E}$, and ornamentation at the polar and equatorial view are the most valuable variables for separating the grape species. The results of UPGMA clustering projection for species are quite common. The results from cluster analysis show that the examined members of the thirty grape cultivars that fall into two main groups coincide with pollen morphological features (Figure 4). According to PCA performed in the thirty grape genotypes, 3 principal components were revealed and they defined $94.98 \%$ of the variance. Pollen width and the length/width ratio had the highest positive contribution to $\mathrm{PC} 1$, constituting $36.68 \%$ of the total variance, which is the most important component. While PC1 is related to pollen width, PC2's association with pores length and pores width are a useful value in differentiation between varieties (Table 5). 


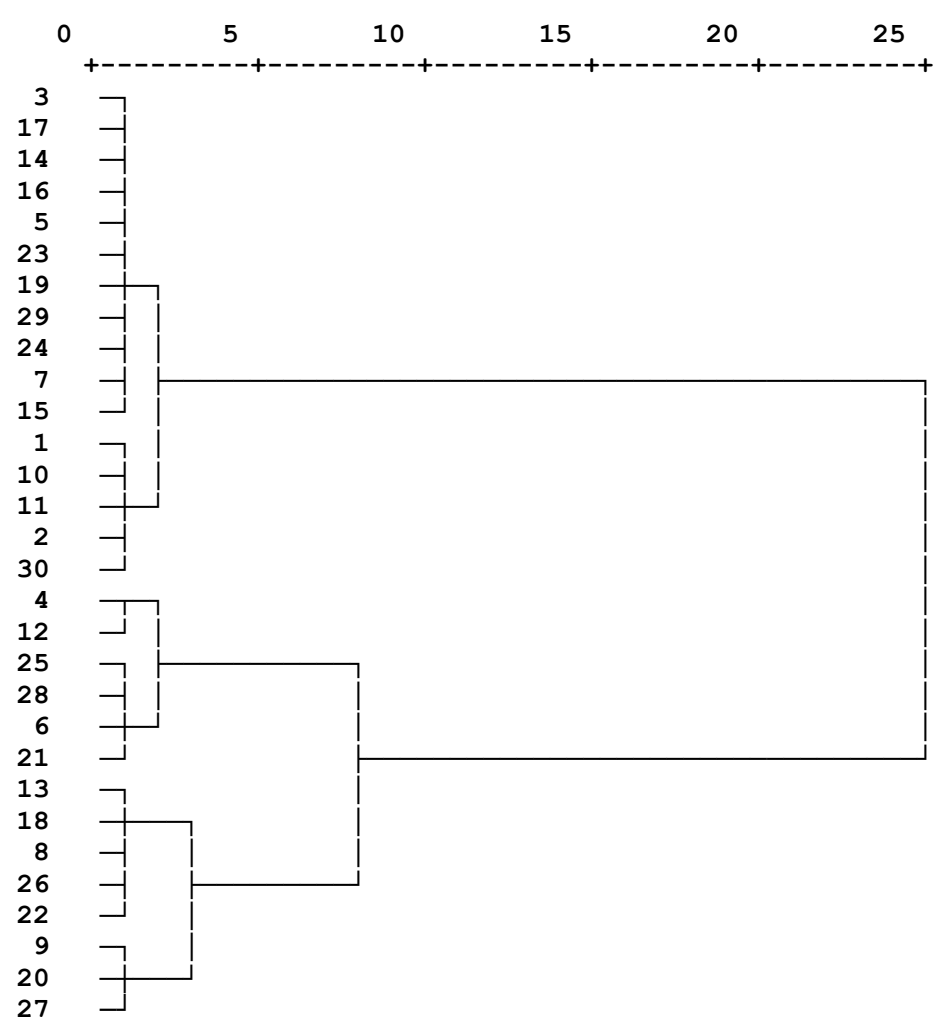

Figure 4- Dendrogram of hierarchical cluster analysis obtained by Ward's clustering method

\section{Conclusions}

In grape species, pollen morphology, exine characteristics, and the presence or absence of pores and furrows can be considered as useful tools for some taxonomic studies.

Morphological characteristics of pollens showed significant differences among the thirty grape cultivars. These properties are the most influential for classification of cultivars into particular groups. A number of palynological investigations into cultivated Vitis varieties also showed that pollen shape and P/E ratio change from one sample to another. The pollen features were found to be the most important parameters for characterization.

The present study confirms the inaperturate and tricolporate pollen grains were observed in cultivars. Some differences in size, polarity and ornamentation were observed among some of the studied cultivars in some cases among the thirty grape cultivar. There were differences in pollen ornamentation in the cultivars studied. In this regard "Alicante Bouschet", "Buca Razakı", "Syrah", "Ohannes", "Morseyhative", "Hafizali", "Cardinal”, "Tarsus Pembesi", "Şika" cultivars were without furrows. On the other hand, areolat pollen determined in some cultivars were, such as "Foça Karası", "Mahrabaşı”, "Yuvarlak Razakı", "Beyaz Şam”, "Müşküle”, "Trakya İlkeren”, “Alphonse Lavallée”, "Siyah Gemre”, "Cinsault”, "Kozak Gemresi”, "Malbec”, "Italia”, “Öküzgözü”, "Pembe Gemre”, "Papaz Karası”, “Kırmızı Şam”, "Morsleleh”, “Abiguş”, "Çeşme Pembesi”, "Pek Üzümü”, "Yuvarlak Çekirdeksiz". These morphological properties of pollen can be used for identification of varieties. Wine grapes and table grapes were distributed among groups. Considering the features examined, the fact that the cultivars can be divided into groups by means of a cluster analysis is an indication that they can be used in the identification of varieties. Palynology of Vitis vinifera L., is an adequate and complementary observation for identification.

\section{Disclosure statement}

No potential conflict of interest was reported by the author.

\section{References}

Ahmedullah M (1983). Morphology of pollen from selected Vitis cultivars. J. Am. Soc. Hortic. Sci. 108: 201-206

Cabello F S Santa Maria, P de Luis Villota, M E Tortosa Tortola (1994). Palynological study of the pollen grain of Vitis vinifera L. cultivars. Some aspects of sculpturing and pollination. Vitis 33(2): 57-61. https://doi.org/10.5073/vitis.1994.33.57-61

Eichhorn K W \& Lorenz H (1977). Phaenologische entwicklungstadien der rebe. Nachrichtenblatt des deutschen pflanzenschutzdienstes (Braunschweig) 29: 119-120

Erdtman G E (1952). Pollen Morphology and Plant Taxonomy. Angiosperms. (An Introduction to Palynology I.). 539 pp. Almquist and Wiksell (Stockholm), USA 
Faegri K \& Iversen J (1989). Textbook of Pollen Analysis. 328 pp. John Wiley \& Sons Ltd., Chichester

Gallardo A R, Ocete R, Ángeles López M, Lara M \& Rivera D (2009). Assessment of pollen dimorphism in populations of Vitis vinifera L. subsp. sylvestris (Gmelin) Hegi in Spain. Vitis 48(2): 59-62

Gökbayrak Z \& Engin H (2016). Micromorphology of pollen grains of some grape cultivars. VII International Scientific Agriculture Symposium “Agrosym 2016”. Book of Proceedings 176-180. ISBN: 9789997663276

Hyde H A \& Adams K F (1958). An Atlas of Airborne Pollen Grains. 116 pp. Macmillan \& Co., Ltd., London

Inceoglu O, Pınar N \& Oybak-Dönmez E (2000). Pollen morphology of wild Vitis sylvestris Gmelin (Vitaceae). Turk. J. Bot 24: 147-150. Corpus ID: 30573633

Jovanovic-Cvetkovic T, Micic N, Djuric G \& Miljan Cvetkovic M (2016). Pollen Morphology and Germination of Indigenous Grapevine Cultivars Žilavka and Blatina (Vitis vinifera L.). AgroLife Scientific Journal, 5(1): 105-109. ISSN ONLINE: 2286-0126

Lombardi J A (2007). Systematic of Vitaceae in South America. Canadian Journal of Botany 85: 712- 721. https://doi.org/10.1139/B07-021

Mabberely D I (1987). The Plant Book. Camb. Univ. Press, Cambridge, New York

Marasalı B, Pınar M \& Büyükkartal N H (2005). Palynological Study on the Pollen Grains of Selected Turkish Grape (Vitis vinifera L.) Cultivars. Turk J Agric For 29: 75-81

Martens M H R, Reisch B I \& Mauaro M C (1989). Pollen size variability within genotypes of Vitis. HortScience, 24: 659-662

Najmaddin C, Hussin K \& Maideen H (2013). Comparative leaf anatomical of selected species in Vitaceae and Leeaceae. American Journal of Applied Sciences 10(4): 414-417. https://doi.or/10.3844/ajassp.2013.414-417

Reille M (1966). Contribution a I'etude palynologique de la famille de Vitaceae. Memoire Diplome Etudes Supereures. 88 pp. Montpellier

Roytchev V (1995). Palynobiometric studies in vines (Vitis vinifera L.). Vitis 34: 197-200

Roytchev V (1997). Palynological Classification of Seedless Grape (Vitis vinifera L.) Cultivars. Am. J. Enol. Vitic 48(2): 207-213

Roytchev V, Terziisky D, Dimova D \& Karageorgiey S (1994). Scanning electron microscopy study of pollen morphology in seedless grape (Vitis vinifera L.) cultivars. Vitis 33: 105-108

Slimane B M \& Askri F (1990). La taille des grains de pollen comme moyen d'identification de souches de vignes tunnisiennes. Bull Organ Int Vigne Vin 63: 899-909

Storey W B (1975). Figs. In: Editors Janick J and Moore JN. Advances in fruit breeding. 568-589

Kharitonashyili L A, Ramishyili R M \& Kipliani M G (1989). Study of the pollen in various types on Vitis vinifera L. flowers using a scanning electron microscope. Soobshch. Akad. Nauk Gruz. SSR 136: 653-656

Tanaka N, Uehara K \& Murata J (2004). Correlation between pollen morphology and pollination mechanisms in the Hydro charitaceae. J Plant Res. 117: 265-276. https://doi.org/10.1007/s10265-004-0155-5

Uzun H I \& Ilter E (1987). Studies on Vitis pollen using scanning electron microscopy. Doga, Türk Tarim Ormancilik Derg., 11: 457-460

Van Der Pluym A \& Hideux M (1977). Application d'une methodologic quantitative á la palynologic d' Eryngiummaritimum (Umbelliferae). Plant. Syst. Evol. 127: 55-85

Wang G, Chen J, Li Z B, Zhang F P \& Yang D R (2014). Has pollination mode shaped the evolution of Ficus pollen? PLoS ONE 9(1): e86231. https://doi:10.1371/ journal pone.0086231

Wodehouse R P (1935). Pollen Grains, Their Structure, Identification and Significance in Science and Medicine. 574 pp. McGraw-Hill Book Company Inc., New York and London

(C) 2022 by the author(s). Published by Ankara University, Faculty of Agriculture, Ankara, Turkey. This is an Open Access article distributed under the terms and conditions of the Creative Commons Attribution (CC BY) license (http://creativecommons.org/licenses/by/4.0/), which permits unrestricted use, distribution, and reproduction in any medium, provided the original work is properly cited. 